\title{
Protocol for Rapid, Accurate, Electrophysiologic, Auditory Assessment of Infants and Toddlers
}

\author{
Yvonne S. Sininger $^{1} \quad$ Lisa L. Hunter ${ }^{2,3}$ Patricia A. Roush ${ }^{4}$ Sue Windmill ${ }^{2}$ Deborah Hayes ${ }^{5,6}$ \\ Kristin M. Uhler ${ }^{5,6}$
}

${ }^{1} \mathrm{C} \&$ Y Consultants, Santa Fe, NM

2 Department of Otolaryngology, Head and Neck Surgery, Cincinnati Children's Hospital Medical Center, Cincinnati, $\mathrm{OH}$

${ }^{3}$ Department of Communication Disorders, University of Cincinnati, Cincinnati, $\mathrm{OH}$

${ }^{4}$ Department of Otolaryngology, Head and Neck Surgery, University of North Carolina Medical Center, Chapel Hill, NC

${ }^{5}$ Department of Pediatrics, Colorado School of Medicine, Denver, CO

${ }^{6}$ Department of Physical Medicine and Rehabilitation, Colorado

School of Medicine, Denver, CO

J Am Acad Audiol 2020;31:455-468.
Address for correspondence Yvonne Sininger, C \& Y Consulting, Santa Fe, NM 87506 (e-mail: ysininger@cnyconsult.com).

\author{
Abstract \\ Keywords \\ - audiology \\ - auditory \\ - auditory brainstem \\ response \\ - auditory steady-state \\ response \\ - auditory threshold \\ - Bayesian weighting \\ - CE-chirp \\ - distortion-product \\ otoacoustic \\ emissions \\ - evoked potentials
}

Background Audiologists often lack confidence in results produced by current protocols for diagnostic electrophysiologic testing of infants. This leads to repeat testing appointments and slow protocols which extend the time needed to complete the testing and consequently delay fitting of amplification. A recent publication (Sininger et $\mathrm{al}^{50}$ ) has shown how new technologies can be applied to electrophysiologic testing systems to improve confidence in results and allow faster test protocols. Average test times for complete audiogram predictions when using new technologies and protocols were found to be just over 32 minutes using auditory brainstem response (ABR) and just under 20 minutes using auditory steady-state response (ASSR) technology.

Purpose The purpose of this manuscript is to provide details of expedited test protocols for infant and toddler diagnostic electrophysiologic testing.

Summary Several new technologies and their role in test speed and confidence are described including CE-Chirp stimuli, automated detection of ABRs using a technique called $F_{\mathrm{MP}}$, Bayesian weighting which is an alternative to standard artifact rejection and Next-Generation ASSR with improved response detection and chirp stimuli. The test protocol has the following features: (1) preliminary testing includes impedance measures and otoacoustic emissions, (2) starting test levels are based on BroadBand CE-Chirp thresholds in each ear, (3) ABRs or ASSRs are considered present based on automated detection rather than on replication of responses, (4) number of test levels is minimized, (5) ASSR generally evaluates four frequencies in each ear simultaneously with flexibility to change all test levels independently.

Conclusions Combining new technologies with common-sense strategies has been shown to substantially reduce test times for predicting audiometric thresholds in infants and toddlers (Sininger et $\mathrm{al}^{50}$ ). Details and rationales for changing test strategies and protocols are given and case examples are used to illustrate.
Copyright $\odot 2020$ by the American Academy of Audiology. All rights reserved. Thieme Medical Publishers, Inc., 333 Seventh Avenue, New York, NY 10001 , USA.

Tel: +1(212) 760-0888.
DOI https://doi.org/ 10.3766/jaaa.19046. ISSN 1050-0545. 


\section{Introduction}

Dramatic improvements in technology have been implemented in the past 5-10 years, allowing for a change in strategies and protocols for obtaining audiometric electrophysiologic assessments of infants and toddlers. These changes will lead to more reliable results and shorter test times. Faster testing means fewer compromises and shortcuts. Information needed to make on-the-spot decisions during testing is also now available to clinicians. This article will describe an approach and test protocol that eliminate guesswork and has been shown to reduce test time in a recent clinical demonstration project (Sininger et $\mathrm{al}^{50}$ ).

Fast and accurate hearing threshold evaluations are needed for infants who do not pass newborn screening, as well as for the thousands of children at risk for hearing loss due to illness and syndromes associated with hearing loss or those at risk because of exposures to medications such as ototoxic medications. Expediting all stages of early identification is critical to achieve the ultimate goal of providing early intervention including fitting of amplification. The importance of this process happening quickly to facilitate communication development has been emphasized repeatedly (Yoshinaga-Itano ${ }^{61}$; Joint-Committee-On-Infant-Hearing ${ }^{36}$; Sininger et $\mathrm{al}^{49}$; Yoshinaga-Itano et $\mathrm{al}^{62}$ ).

According to the 2007 statement of the $\mathrm{JCIH}$, diagnostic electrophysiologic evaluation of infants who fail screening should be completed by three months of age. New information on the importance of early intervention may influence the JCIH to recommend, in the near future, that the diagnostic evaluation be completed even earlier. Given that the current 1-3-6 guidelines are often not met (Yoshinaga-Itano et $\mathrm{al}^{62}$ ), meeting even more stringent guidelines will require dramatic changes in audiologic procedures.

The diagnostic test is necessary to proceed with rehabilitation and requires an electrophysiologic methodology for which the infant must sleep during the assessment $\left(\mathrm{JCIH}^{36}\right.$; Dworsak-Dodge et $\mathrm{al}^{17}$ ). Test sessions in which the infant sleeps naturally are preferred because they avoid sedation or anesthesia and generally can be accomplished with proper scheduling in infants younger than three months. However, if the process is not concluded by three months of age because the testing could not be completed in a single appointment or clinicians are not confident in results, the consequences generally include multiple repeat appointments and often the need to sedate or anesthetize the child for testing. This adds cost, additional parental anxiety, scheduling delays, and other complications. Smaller audiology clinics may not have the facility to provide proper, medically supervised anesthesia requiring an outside referral. Added to the difficulties of delayed diagnosis are potentially missed appointments and/ or the total loss to follow-up, increased parental anxiety, increased audiology workloads, and others. Of course, delay in the child's treatment and communication development is by far the most serious outcome of delays in the early hearing detection and intervention process.

A recent study by Sininger et $\mathrm{al}^{50}$ found that half of the infants and toddlers requiring an electrophysiologic audio- metric evaluation in their clinical sample had normal hearing. Although the mission of newborn hearing screening is to identify, characterize, and habilitate hearing loss, those children who did not pass screening at birth and yet do not have permanent hearing loss also need to be accurately identified and released from the clinical process in a timely manner.

One obvious solution to late diagnosis is to obtain a complete, accurate audiogram prediction and additional diagnostic information during the first, postscreening, audiology appointment when the infant should be younger than three months and can be tested in a natural sleep state. This goal requires protocols and technology that will facilitate rapid and accurate testing. According to Janssen et $\mathrm{al}^{34}$, the average sleep time during an evaluation for infants around four months of age is 48.8 minutes, but $20 \%$ of the infants evaluated slept for 33.1 minutes or less. A full diagnostic assessment should include audiometric thresholds in each ear at four frequencies $(500,1000,2000$, and $4000 \mathrm{~Hz}$ ), eight thresholds in total. Janssen et al found that they were able to obtain only four thresholds in $80 \%$ of cases in a natural sleep session in which they were using standard auditory brainstem response (ABR) methodology with tone burst stimuli.

Sininger et $\mathrm{al}^{50}$ demonstrated that predicted auditory thresholds at four frequencies in a clinical sample of infants and toddlers by automated detection of ABR and nextgeneration auditory steady-state response (ASSR) were equal or slightly lower by ASSR. Excellent agreement in thresholds for the two techniques provides validation of the automated detection algorithms as well as the test protocols used to obtain thresholds for both techniques. The $r^{2}$ values for the ABR and ASSR thresholds measured at 500, 1000, 2000 and $4000 \mathrm{~Hz}$ ranged from 0.852 to 0.956 . These compare favorably with other similar validation studies, where ABR thresholds were compared with behavioral thresholds (Stapells et $\mathrm{al}^{52}$ ) with equivalent $r^{2}$ values of 0.88-0.94 or ABR compared with ASSR (Van Maanen and Stapells ${ }^{59}$ ), which found equivalent $r^{2}$ values ranging from 0.59 to 0.79 . In both these studies, the correlations were reported as $r$ and the aforementioned values are the squared equivalent.

The average test times, achieved in the Sininger et $\mathrm{al}^{50}$ study, are exceptionally fast. Four thresholds in each ear could be predicted at an average of 32.15 minutes using automated ABR and at 19.93 minutes using new ASSR technology. Test times were found to be even faster in cases of normal hearing (24.62 and 15.31 minutes on average for ABR and ASSR, respectively). Achieving these test times required specific procedures, including specialized stimuli, response detection technologies, and time-saving protocols. The roles of the stimuli and response detection algorithms in reducing test times are discussed thoroughly in the original article (Sininger et $\mathrm{al}^{50}$ ) and in later sections here. The purpose of this article is to fully describe the specific test procedures, to give rationales, and to illustrate these with case studies. Without the implementation of our specific test protocol, the fast test times demonstrated would not be possible. 


\section{Saving Time and Improving Accuracy}

Some factors are not easily changed. Natural-sleep nap durations are not under the direct control of the clinician but can be optimized. Clinicians should develop a careful sleep deprivation plan that, if followed by the families, will increase the likelihood and duration of sleep. Infants should be dry and fed just before testing to sleep more quietly and for a longer time. Swaddling can be a very effective way to comfort infants and help induce sleep. Swaddled infants should be monitored to be sure that the blanket does not come loose (www.healthychildren.org/English/ages-stages/baby/sleep/Pages/A-ParentsGuide-to-Safe-Sleep.aspx). The fact remains that these techniques are not foolproof, and the duration of the natural-sleep nap is limited. One can expect about 48 minutes of sleep for infants younger than three months (Janssen et $\mathrm{al}^{34}$ ).

\section{New Technologies}

Sleep time is limited, but technology can be applied to improve speed, accuracy, and consistency of pediatric electrophysiologic evaluations. Two technologies have been particularly useful in this regard. First, specially engineered stimuli (chirps) will produce larger amplitude electrophysiologic responses which are more easily detected in less time than standard click or tone burst stimuli. Second, real-time feedback to the tester on the quality of recordings and accurate statistical detection of responses will maximize the use of test time and improve the accuracy of threshold measurements.

\section{CE-Chirps}

Recently described wideband (WB) (Cebulla et $\mathrm{al}^{8}$; Elberling and Don $^{21}$; Cebulla and Elberling ${ }^{4}$ ) and narrowband stimuli (Sturzebecher et $\mathrm{al}^{58}$ ), known as CE-Chirps, have the distinct advantage of producing a neural response that is larger in amplitude than the potential generated by standard (click or tone burst) stimuli under the same recording conditions. When using these stimuli, the average noise needs less reduction to reveal the (larger) response and, thus, the averaging (test) time is reduced.

CE-Chirp stimuli produce larger responses than traditional stimuli because the time of arrival of low- and high-frequency energy components in the stimulus is staggered in a manner that counteracts the frequency-dependent time delay imposed by the basilar membrane. Consequently, low-, middle-, and high-frequency auditory neurons are activated simultaneously, avoiding response phase cancellations across frequencies and maximizing neural synchrony and, subsequently, the amplitude of the composite neural activity.

The response amplitude advantage afforded by CE-Chirps as compared with traditional clicks and tone bursts or pips has been well documented and ranges from $30 \%$ to $100 \%$ (Elberling and Don ${ }^{21}$; Ferm et $\mathrm{al}^{27}$; Cebulla et $\mathrm{al}^{5}$; Cho et $\mathrm{al}^{9}$; Ferm and Lightfoot ${ }^{26}$; Cobb and Stuart ${ }^{10,11}$ ). The use of CEChirp stimuli consequently improves the speed and positive rate of response detection (Stürzebecher et $\mathrm{al}^{58}$; Cebulla et $\mathrm{al}^{8}$; Stuart and $\mathrm{Cobb}^{54}$ ) and lowers the threshold in $\mathrm{dB}$ $\mathrm{nHL}$ of the response (Ferm et $\mathrm{al}^{27}$; Ferm and Lightfoot ${ }^{26}$; Michel and Jorgensen ${ }^{41}$ ).
More than a decade of work has gone into the development of the CE-Chirp (Cebulla et $\mathrm{al}^{8}$; Elberling et $\mathrm{al}^{23}$; Elberling and Don ${ }^{21,22}$; Cebulla and Elberling ${ }^{4}$; Elberling et $\mathrm{al}^{18}$; Elberling et $\mathrm{al}^{24}$; Kristensen and Elberling ${ }^{38}$ ), and the result is a clinical tool with published accurate and reliable results in infants and older children. A change in the original model which incorporates the stimulus level into the equation for the generation of CE-Chips has added a delay compensation needed to account for performance differences based on the stimulus level (Elberling and Don $^{22}$ ). Kristensen and Elberling ${ }^{38}$ have shown that these "levelspecific" or level specific (LS) CE-Chirps demonstrate a consistent amplitude advantage over the click at all stimulus levels and also demonstrate better resolution of all peaks of the ABR waveform. Narrowband LS CE-Chirp stimuli have been implemented for both ABR and ASSR assessments and are recommended here as used in the Sininger et $\mathrm{al}^{50}$ study.

\section{$F_{\mathrm{MP}}$ for Response Detection of ABR}

Objective response detection is the key to optimizing test time in audiometric application of electrophysiology. An optimal system would stop averaging when either a response is detected or, in the absence ofan answer, when the noise is sufficiently low to allow detection if a response was present. In this case, a "no response" is justified. When compared with using a fixed number of sweeps, this stopping rule will save test time when the ABR is large and/or background noise is low, but will apply adequate averaging time to resolve low amplitude (near threshold) responses or those with high noise. Thus, averaging time is used efficiently.

For the $\mathrm{ABR}$, the $F_{\mathrm{SP}} / F_{\mathrm{MP}}$ response detection algorithm meets these criteria. Described initially more than 30 years ago (Don et $\mathrm{al}^{16}$; Elberling and Don ${ }^{19}$ ), the method has been published repeatedly in experiments to characterize response threshold (Elberling and Don $^{20}$; Cone-Wesson and Ramirez $^{12}$; Sininger et $\mathrm{al}^{48}$ ), to describe the nature of background noise during averaging (Don and Elberling ${ }^{14}$ ), to determine when to stop averaging (Don and Elberling ${ }^{15}$ ), and as an aid to clinical testing (Sininger ${ }^{46}$ ). In 1998, Hyde et al said, "One of the most thoroughly researched methods to date, the variance ratio $F_{\mathrm{SP}}$ can significantly improve the objectivity and consistency of ABR measures" (Hyde et $\mathrm{al}^{33}$, p. 112).

$F_{\mathrm{MP}}$ are values that describe the "quality" of the response and are closely related to the signal-to-noise ratio. The noise in the average response is estimated by calculating the variance of the amplitude of either a single point $\left(F_{\mathrm{SP}}\right)$ or multiple (5) points $\left(F_{\mathrm{MP}}\right)$ per sweep for approximately 256 points. Large variance in amplitude from sweep to sweep is clear evidence of a noisy recording and vice versa. With $F_{\mathrm{MB}}$ the estimated background noise in the average is quantified and reported at regular intervals. The numerator of the $F_{\mathrm{SP}} / F_{\mathrm{MP}}$ equation is the amplitude variance across a central 10 -msec period of the average (taken at regular intervals, every 50 sweeps for $F_{\mathrm{MP}}$ ). Consider that a very low variance would be indicative of flat average found when ABR amplitude is either very small or no response exists. The variance across the response window will always include any ABR (signal) and unresolved noise. It is, 
therefore, possible to have high residual noise in the recording contributing to a high variance in the numerator, regardless of the amplitude of any ABR. Therefore, the $F_{\mathrm{SP}} / F_{\mathrm{MP}}$ must divide the numerator by the estimated background noise (Elberling and $\operatorname{Don}^{19}$ ) and the resulting value is related to the true signalto-noise ratio (SNR) plus one. ( $F_{\mathrm{MP}}$ numerator is the [Signal $\{A B R\}+$ Noise], whereas the denominator is [Noise]. Signal + Noise $/$ Noise $=$ SNR +1 .)

The $F_{\mathrm{MP}}$ and the background noise functions are plotted during the averaging process, providing valuable information to audiologists (see - Figure 1). The noise function is reduced logarithmically as the number of averaged sweeps increases (Don and Elberling ${ }^{14}$ ). The amount of averaging needed to achieve a given noise level that will produce evidence of a response will depend on the stability and level of the noise as well as on the overall amplitude of the ABR. It should be noted that the reduction in noise is responsible for the growth of the $F_{\mathrm{MP}}$ function. Reduction in noise with time is the sign of a stable average, and, conversely, persistent high noise is the sign of poor recording conditions and should be investigated.

The value of $F_{\mathrm{MP}}$ is updated every 50 sweeps and, if a response is present, grows as the noise is reduced with averaging, as shown in the example in - Figure 1. The $F_{M P}$ as a ratio of variances, has an $F$ distribution with 5 and 250 degrees of freedom (Elberling and Don ${ }^{19}$ ), which allows the determination of confidence levels for $F_{\mathrm{MP}}$ values. The $99 \%$ confidence level of a true response presence is $F_{\mathrm{SP}}$ or $F_{\mathrm{MP}}=3.10$, and the $95 \%$ confidence level is 2.25 . Sininger ${ }^{46}$ found that the $95 \%$ confidence of $F_{\mathrm{MP}}$ correlates best with the "visual response detection" of a trained clinician. Consequently, the recommended stopping value for $A B R$ response detection is $F_{\mathrm{MP}}=2.25$ after a minimum of 800 sweeps.

If no response is detected, it is efficient to stop averaging when the background noise has been reduced sufficiently to have allowed detection of a small response if it were present. The Sininger et $\mathrm{al}^{50}$ study evaluated ABR in pediatric patients

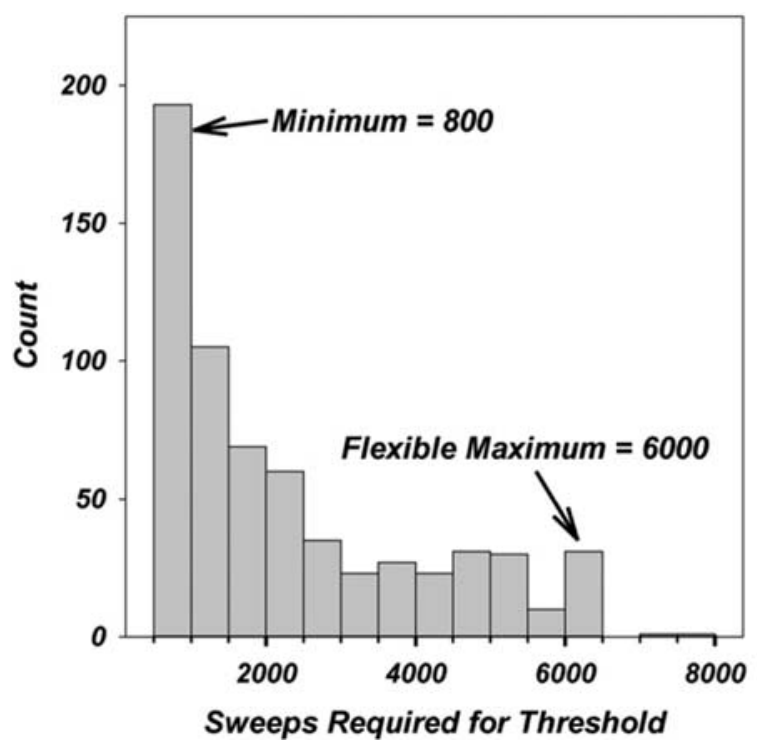

Fig. 2 Distribution of the number of sweeps required to reach the ABR threshold for all stimuli, both ears, and all participants. Eight hundred was the minimum number of sweeps allowed, and for most averages, 6,000 was the maximum. The user could extend the maximum number of sweeps as needed.

with NB CE-Chirps from 500 to $4000 \mathrm{~Hz}$ using $F_{\mathrm{MP}}$ as the response criteria. Based on that study, a 15-nV noise level is recommended for stopping an average in a "no response" condition.

The distribution of the number of sweeps needed to reach the $2.25 F_{\mathrm{MP}}$ criteria at threshold in the Sininger et al $^{50}$ study is shown in -Figure 2. As can be seen, there is an objective stopping rule demonstrating that responses can be adequately resolved even at threshold, with as few as 800 sweeps, but others may be low amplitude and/or high noise and require as many as 8,000 sweeps to be determined. Any chosen value for a "fixed" number of sweeps for averaging would be inadequate and would either average longer than necessary when the

\section{$F_{\mathrm{MP}} /$ Residual Noise Plot}
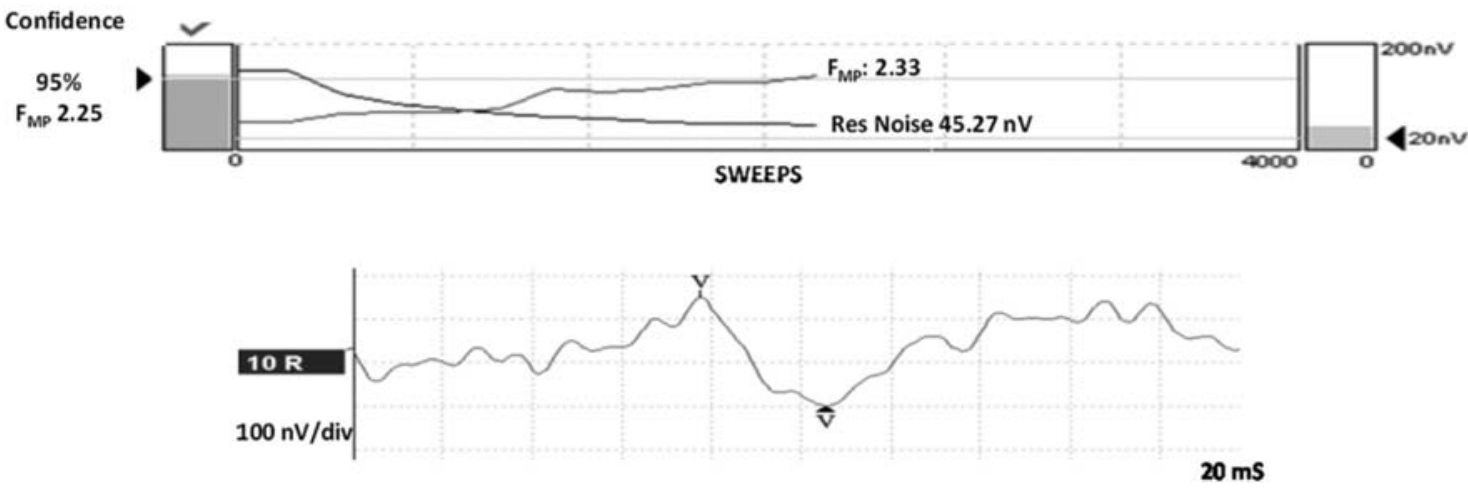

Fig. 1 Plot of $F_{M P}$ and residual noise by averaged sweeps for the ABR shown below elicited by a $10 \mathrm{~dB} n \mathrm{HL}$ WB CE-Chirp in an infant. The plot at the top is $F_{M P}$. The recording was stopped at about 2,500 sweeps when the $F_{M P}$ exceeded 2.25 which is the $95 \%$ response confidence level shown at the left. The indicator box turns green when a response is reached and, if selected, the average is terminated. The descending plot is the residual noise which achieved a value of $45.27 \mathrm{nV}$ when the recording was terminated by $\mathrm{F}_{\mathrm{MP}}$. The $20 \mathrm{nV}$ noise value at the right is the set stopping criteria for background noise for this recording. The $F_{M P}$ and the residual noise level should both be set, and the first to reach criterion value before the maximum number of sweeps is achieved will stop the recording. 
response is large or not average long enough to see small responses at threshold. Based on the data in - Figure 2, the recommended maximum number of sweeps for ABR averages is 6,000 . The average will usually be stopped before that time but can be increased by the user in individual cases.

\section{Bayesian Weighting}

The process known as "Bayesian weighting" was first proposed by Elberling and Wahlgreen ${ }^{25}$ as a method of noise management during averaging. Rather than rejecting all sweeps with high amplitude (traditional noise rejection), Bayesian weighting assigns a "weight" to each block (50) of sweeps based on the estimated noise in the block (multipoint variance as calculated for $F_{\mathrm{MP}}$ ). All blocks of sweeps are included in the average, but each has a weighting factor determined by its background noise. A low noise block is given greater "weight" in the overall average, and the noisy block of sweeps is assigned less weight in the average, but no data are discarded. Large bursts of noise that sometimes contaminate otherwise quiet recordings can be minimized because they receive low weights in the average (Don and Elberling ${ }^{14}$ ).

Lightfoot and Stevens ${ }^{39}$ compared the effects on residual noise of standard artifact rejection (AR) levels and Bayesian weighting techniques in babies. Bayesian weighting was implemented along with a $10-$ and $20-\mu \mathrm{V}$ standard AR. Each average had an acquisition time of 61 seconds. They found that in low noise conditions, standard AR of $5 \mu \mathrm{V}$ produced the lowest residual noise, but Bayesian weighting with $10 \mu \mathrm{V}$ produced nearly equivalent results. For moderate noise conditions, a $6.5-\mu \mathrm{V}$ standard AR and Bayesian weighting with a $10-\mu \mathrm{VAR}$ produced averages with equivalent noise levels and in severe noise level conditions, the Bayesian weighting $(10 \mu \mathrm{V} A R)$ produced averages with the lowest noise. Because infants' noise levels during testing may not be obvious at the beginning of a recording, it would be prudent to use the Bayesian weighting with the $10-\mu \mathrm{V}$ standard $\mathrm{AR}$ that will provide the most help if the infant is very noisy and essentially equivalent results if the noise levels are mild or moderate.

\section{Automated Detection of ASSR}

A tonal stimulus that is amplitude-modulated at a specific frequency should produce a spike in the spectrum of the EEG at that frequency. Detection of ASSR may involve inspection of the phase of the neural response at the modulation frequency for "phase coherence" and/or the magnitude of the spectral component at the modulation frequency, which is compared with surrounding frequencies with an $F$-test (Korczak et $\mathrm{al}^{37}$ ). These tests are termed "single sample" tests as they use information in the first harmonic (modulation) frequency only. Cebulla et $\mathrm{al}^{7}$ demonstrated the advantages of using information from as many as six harmonics of the modulation frequency for the detection with ASSR. That study compared several statistical approaches with a single sample and qsample tests and found that the six sample test produced higher detection rates and reduced detection times.

Bonferroni corrections for multiple statistical tests were replaced by more appropriately corrected critical test values developed with a simulation procedure (Sturzebecher et $\mathrm{al}^{57}$ ). The Eclipse ASSR system implements this procedure with a table lookup of critical test values "for each test step number that corresponds exactly to the given error probability" (Sturzebecher and Cebulla ${ }^{56}$, p. 862). This method allows individualized statistical criteria for ASSR tests based on the exact number of test steps used, further improving detection rates and decreasing test time (Sturzebecher and Cebulla $^{56}$; Cebulla and Sturzebecher ${ }^{6}$ ). The current Eclipse ASSR detection scheme, which we will term "next generation," includes assessment of amplitude and phase in 12 modulation harmonics. The excellent sensitivity of this ASSR system was demonstrated by the ability to detect thresholds at levels as low or lower than good ABR technology in normal-hearing infants and toddlers (Sininger et $\mathrm{al}^{50}$ ).

\section{Avoid Unnecessary Tests}

Without an objective measure of response presence or absence, the audiologic community has promoted the testretest principle. Consequently, ABRs, even those that seemed to contain an obvious response or clear absent response, are often repeated at a given stimulus level to check for consistency. This repetition has the potential of doubling the test time and is unnecessary when better methods of response validation, including common sense, are available. Also, when doing a "threshold search" procedure, it is unnecessary to routinely test at regular stimulus intervals. In other words, there is no real need for a latency-intensity function to be produced when time is limited. Our protocol also describes ways to predict the sensation level of the stimulus from the characteristics of the response, which can guide in the selection of subsequent stimulus levels.

\section{Multiple Tests and Test Order}

Years ago, Jerger and Hayes ${ }^{35}$ described the cross-check principle for audiology. They emphasized the need to validate the findings of one test by looking for consistency in additional tests. In that same vein, the order of test presentation can serve to expedite the test battery, which in this instance is critically important. The test battery described strongly suggests that testing begins with tympanometry (WB and three dimensional if possible) and otoacoustic emissions. These tests can generally be performed very quickly, do not require that the child is sleeping, and often can be performed on the same equipment for expediency. Whereas they should not be performed in isolation, prior knowledge of the status of the middle ear and the presence or absence of otoacoustic emissions can be very valuable when attempting to focus in on whether hearing loss is present and, if so, what is the type of loss. Both tests have been shown to be valuable, particularly in predicting conductive hearing loss (Hunter et $\mathrm{al}^{32}$; Prieve et $\mathrm{al}^{44}$; Aithal et $\mathrm{al}^{1}$; Blankenship et $\left.\mathrm{al}^{3}\right)$.

The protocol being described also suggests using a WB CEChirp stimulus for a quick, preliminary threshold test by ABR in each ear. This threshold will indicate appropriate starting levels for frequency-specific threshold searches when using either ABR or ASSR tests. 


\section{Protocol}

Pre-electrophysiologic Measures: Tympanometry and Distortion Product Otoacoustic Emissions (DPOAEs)

The Sininger et $\mathrm{al}^{50}$ study used the following procedures that worked effectively and thus are recommended. However, the study emphasized both ABR and ASSR methods. All tests were performed on the Interacoustics Eclipse system. The software version for ABR (EP25) was 4.2.1 and for ASSR was 1.2.6. The exact methodology described here for tympanometry and otoacoustic emissions has not been optimized to achieve the best results, but the performance of these tests relative to the entire test battery will be discussed.

WB tympanometry used medium pump speed, a pressure range of +200 to -400 daPa, and a click stimulus to elicit the response. The WB response was initially plotted in three dimensions: pressure by frequency by absorbance. Narrowband $(800-2000 \mathrm{~Hz})$ and singlefrequency tympanograms $(226$ and $1000 \mathrm{~Hz}$ ) were extracted and displayed along with normative data based on the child's age. Absorbance was also plotted by frequency overlaid on age-specific normative areas. Visual inspection of the narrowband tympanometry and the absorbance plot relative to normative data for the child's age were used to categorize the ear's test as normal or abnormal (Hunter et $\mathrm{al}^{32}$; Hunter et $\mathrm{al}^{31}$; Hunter et $\mathrm{al}^{29,30}$; Aithal et $\mathrm{al}^{2}$; Myers et $\mathrm{al}^{42,43}$ ).

DPOAEs were evaluated using the Interacoustics Titan with the same probe mechanism used for WB tympanometry. DPOAE SNR was measured with $f 2$ of $2,3,4,5,6$, and $8 \mathrm{kHz}, f 2 / f 1=1.22$, and stimulus levels of 65 and 55 (L1 and L2) dB SPL. The criteria for a response at each frequency included a min imum DPOAE level of $-10 \mathrm{~dB}$, SNR of $6 \mathrm{~dB}$, and residual noise of $-20 \mathrm{~dB}$ SPL or less. It should be noted that these parameters were not tested for their predictive value in any way as this was not a research goal for the study.

These preliminary tests can be performed quickly on an awake infant, and current testing routines should take no more than 10 minutes. The entire study protocol in Sininger et $\mathrm{al}^{50}$ was lengthy because both ABR and ASSR were evaluated. However, of 102 participants, tympanograms were completed on 80 children in the right ear and on 79 in the left. DPOAEs were completed in 91 right ears and 89 left ears. This indicated that there was ample time and participant compliance to complete these preliminary tests in most clinical participants, especially if only one threshold procedure (ABR or ASSR) is then performed.

Electrode placement should follow the OAE and tympanometry tests to avoid upsetting the infant before the tests. After the preparation for the electrodes and perhaps a feeding and diaper change, the infant is usually ready for sleep. Standard electrode application procedures are suggested with either mastoid or earlobe electrodes, and high midline forehead reference. Ground electrodes can be placed according to the audiologists' preference. Interelectrode impedance should be measured with an emphasis on closely matched impedance across electrodes and all values less than $3 \mathrm{k} \Omega$ if possible. Good electrode preparation is considered essential for quiet recordings, which will ultimately reduce the test time.

\section{Electrophysiologic Measures: ABR and ASSR}

Procedures are described for frequency-specific ABR and ASSR thresholds. It is not necessary to do both tests (as described here), as they have been shown to be essentially equivalent (Sininger et $\mathrm{al}^{50}$ ).

For tests conducted during natural sleep, infants should generally be sleep deprived or tested during their regular nap time. Just before the evaluation, the infant may be fed and the diapers changed as appropriate. Both insert earphones should be inserted securely before the testing begins to avoid disturbing the baby during the test. Natural sleep testing should take place in a sound-isolated room.

Before starting frequency-specific testing, an ABR threshold search using a WB CE-Chirp is recommended. A broadband CE-Chirp threshold in each ear may not predict the entire audiogram but does quickly provide an excellent "starting" level reference for frequency-specific testing, for ABR or ASSR. The appropriate level to use when starting a threshold search is a frequently asked question in audiology. Using the obtained WB CE-Chirp threshold level or perhaps 10 or $20 \mathrm{~dB}$ above, to begin testing with NB stimuli, helps to avoid waking the infant with a loud stimulus. The WB CEChirp threshold will also be able to provide a quick estimate of the average hearing levels in each ear for comparison. The preliminary procedure may start in either ear and should use a quick bracketing with a final 10-dB step. Both ears should be evaluated, and there is no need to search less than 10 or $20 \mathrm{~dB}$ nHL.

\section{ABR-Recommended Threshold Procedures}

\section{Stimuli}

The recommended stimuli used for audiogram prediction are NB CE-Chirps, LS with center frequencies of 500, 1000, 2000, and $4000 \mathrm{~Hz}$ developed by Sturzebecher et al. ${ }^{58}$ Narrow (octave) band chirps were created by the addition of individual cosines, with the phase of each component adjusted to compensate for cochlear delay based on the model of de Boer. ${ }^{13}$ The stimuli are presented at $37.9 \mathrm{~Hz}$ with alternating polarity.

The order of presentation of the four stimuli is at the discretion of the tester. If one ear shows a lower WB CE-Chirp threshold, testing could begin in that ear so that masking could be applied to the opposite ear if necessary. The starting level for all frequencies is determined by adding $0-20 \mathrm{~dB}$ to the WB CE-Chirp threshold. This rule is ear specific.

\section{Recording Parameters}

The recording filter used in the study set low pass at $1500 \mathrm{~Hz}$ and high pass at $100 \mathrm{~Hz}(12 \mathrm{~dB} /$ octave). Although significant ABR energy is found less than $100 \mathrm{~Hz}$ in infants (Spivak ${ }^{51}$; Stuart and Yang ${ }^{55}$; Sininger ${ }^{47}$ ), the $100-\mathrm{Hz}$ high-pass filter was used for several reasons. First, the audiologists were familiar with viewing ABRs recorded with this setting. Second, sedated recordings often took place in hostile electrical noise environments where it was necessary to avoid $60 \mathrm{~Hz}$ contamination. Finally, the $F_{\mathrm{MP}}$ calculations require data to be filtered with $100-\mathrm{Hz}$ high pass. Any filter settings can be used for data acquisition, but before $F_{\mathrm{MP}}$ calculations, 


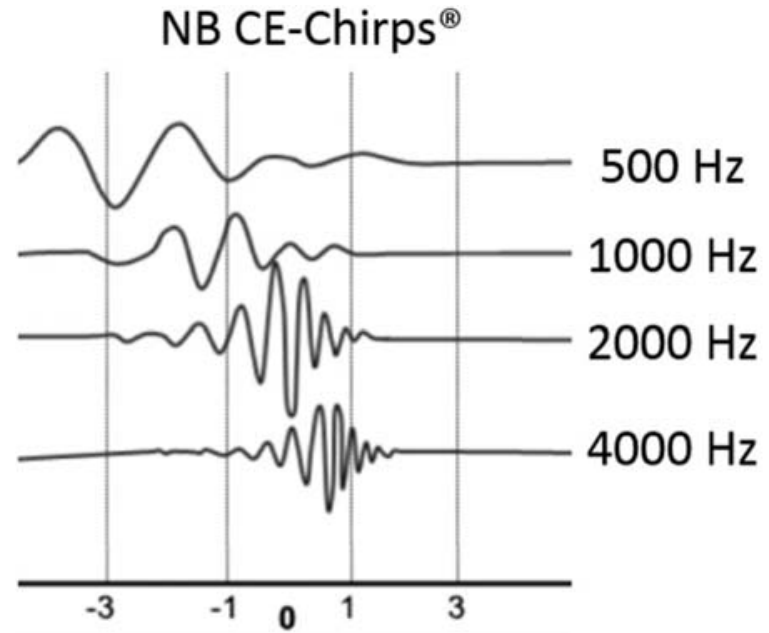

Fig. 3 The time waveforms of the four NB CE-Chirps are shown. The time scale is in ms relative to the onset of the recording window. The presentation of the NB CE-Chirps is staggered relative to the start of the recording window to compensate for the cochlear delay time for each frequency in the same way these components are staggered in the broad-band CE-Chirp. The result is that the expected latency for any of the NB CE-Chirp ABR will be the same as that of the broadband response at the same $\mathrm{nHL}$. Consequently, no changes in the recording window are needed to compensate for expected response latency differences.

the obligatory $100-\mathrm{Hz}$ high pass will automatically be imposed by the software.

Bayesian weighting should be used along with $\mathrm{a} \pm 40 \mu \mathrm{V}$ AR.

The recommended recording window is $20 \mathrm{msec}$. It should be noted that the NB CE-Chirps are staggered in time of presentation based on the known cochlear delay time that eliminates the need for long recording windows when testing with lower frequency stimuli (see - Figure $\mathbf{3}$ ).

\section{Objective Threshold Determination with $\boldsymbol{F}_{\mathrm{MP}}$}

- The stopping rules for averaging are as follows: (a) response confidence of $95 \%\left(F_{\mathrm{MP}} \geq 2.25\right)$ or $(\mathbf{b})$ residual Noise $\leq 15 \mathrm{nV}$ (infants and toddlers). By using both rules, the recording will stop on the target ( $F_{\mathrm{mp}}$ or low noise) that is first reached. The $15 \mathrm{nV}$ noise criterion is only necessary when a "no response" average is being evaluated. The noise can be higher if the $F_{\mathrm{MP}}$ value indicates a response. The number of sweeps should be set high, e.g., 6,000 . Only when neither target is reached will 6,000 sweeps be needed. Note: If the $F_{\mathrm{MP}}$ is growing as the maximum number of sweeps is approached, the number of sweeps can be increased by the tester to improve the chances of reaching the $F_{\mathrm{MP}}$ criteria.

- When the average is completed, repetition at the same level is not needed. Instead, use standard bracketing rules to determine the next level with the following added guidance. If the previous average shows a response present after 1,000 sweeps or less, decrease the level by 10 or $20 \mathrm{~dB}$. If the previous average requires 3,000 or more sweeps to reach the criteria, decrease the level by 5-10 dB.

- If the average stops on "low noise" or at maximum sweeps, increase the level for the subsequent average unless the "maximum level" has been reached. The maximum level of stimulation should be determined at each facility.

- The final step size should be between 5 and $10 \mathrm{~dB}$ at the discretion of the tester. Keep in mind that the added speed found when using these procedures may allow sufficient time to use a 5-dB step.

- The threshold level will be the lowest level demonstrating a positive response $\left(F_{\mathrm{MP}} \geq 2.25\right)$ with a "no response" at the level one step below.

- Correction factors for NB CE-Chirp-generated ABRs are $15 \mathrm{~dB}$ at $500 \mathrm{~Hz}, 10 \mathrm{~dB}$ at $1000 \mathrm{~Hz}$, and $5 \mathrm{~dB}$ at 2000 and $4000 \mathrm{~Hz}$ (see - Table 1). Searching for responses with stimuli below these levels would not be clinically useful. However, given the speed of this technique, including the added amplitude afforded by the CE-Chirp stimuli, there is no need to stop at suprathreshold $(25-30 \mathrm{~dB})$ levels. The Sininger et $\mathrm{al}^{50}$ study did not test less than $20 \mathrm{~dB}$ at $500 \mathrm{~Hz}$ or $10 \mathrm{~dB}$ at the other three frequencies. This allowed a precise prediction ofnormal hearing, while maximizing efficiency.

- Mark peak latencies and amplitudes on an ABR only during the acquisition of another average or after the test. Avoid using precious sleep time to mark peaks.

Table 1 Correction Factors Applied to Thresholds at Dial Reading for Children Tested with ER-3A Insert Transducers

\begin{tabular}{|c|c|c|c|c|c|c|c|c|c|c|c|c|}
\hline \multirow[t]{2}{*}{ Test } & \multirow{2}{*}{$\begin{array}{l}\text { nHL level in } \\
\mathrm{dB}->(\mathrm{Hz})\end{array}$} & \multicolumn{11}{|c|}{$\mathrm{dB} \mathrm{nHL}$ to $\mathrm{dB}$ eHL Correction Factors } \\
\hline & & $0-5$ & $10-15$ & $20-25$ & $30-35$ & $40-45$ & $50-55$ & $60-65$ & $70-75$ & $80-85$ & $90-95$ & 100 \\
\hline \multirow[t]{4}{*}{ ABR eHL } & 500 & -15 & -15 & -15 & -15 & -10 & -10 & -5 & -5 & -5 & 0 & 0 \\
\hline & 1000 & -10 & -10 & -10 & -10 & -5 & -5 & 0 & 0 & 0 & 0 & 0 \\
\hline & 2000 & -5 & -5 & -5 & -5 & -5 & -5 & 0 & 0 & 0 & 0 & 0 \\
\hline & 4000 & -5 & -5 & -5 & -5 & -5 & -5 & 0 & 0 & 0 & 0 & 0 \\
\hline \multirow[t]{4}{*}{ ASSR eHL } & 500 & -25 & -25 & -25 & -25 & -20 & -15 & -15 & -10 & -5 & -5 & 0 \\
\hline & 1000 & -15 & -15 & -15 & -15 & -10 & -10 & -10 & -5 & -5 & 0 & 0 \\
\hline & 2000 & -5 & -5 & -5 & -5 & -5 & -5 & -5 & 0 & 0 & 0 & 0 \\
\hline & 4000 & -5 & -5 & -5 & -5 & -5 & -5 & -5 & 0 & 0 & 0 & 0 \\
\hline
\end{tabular}

Note: ASSR corrections apply to modulation rates at or near $90 \mathrm{~Hz}$ from Interacoustics based on data from Rodrigues and Lewis. ${ }^{45}$ Correction factors diminish with increasing $\mathrm{nHL}$ as suggested by McCreery et al. ${ }^{40}$ 
For all averages, watch the ongoing EEG on the screen. If EEG or residual noise is very high, check electrodes and the baby's state and correct accordingly. Use the pause function if necessary, but the need for pausing is greatly reduced by using the Bayesian weighting. Avoid the temptation to stop the average manually unless high noise is encountered at the beginning of the average. If so, throw away the average and start again. The $F_{\mathrm{MP}}$ graph at the top will turn green, and the averaging will stop if a significant response is reached (see -Figure 1). If no response is found, the system will also stop if the noise goes less than the $15-\mathrm{nV}$ criteria or if the maximum number $(6,000)$ of sweeps are reached. In the meantime, the noise function should descend as the average progresses and the $F_{\mathrm{MP}}$ function should ascend (if a response is present) or may be flat if no response is present. Also, note that if the noise is very high and not diminishing, the $F_{\mathrm{MP}}$ plot will not grow, regardless of whether a response is present. Testers should avoid making judgments about response presence or absence if noise is very high. In this case, a correction to recording conditions should be made to bring the noise into the range that can allow testing.

Sininger et $\mathrm{al}^{50}$ had a simplified approach to bone conduction. This was because of the added test time needed for the research protocol. Sininger recommended the use of a WB CE-Chirp stimulus to obtain a bone conduction threshold by $A B R$ if the tester deemed it necessary. The bone conduction threshold could be compared with the WB CE-Chirp air conduction thresholds. Based on previous studies that find air-bone gaps in normal-hearing babies particularly at $500 \mathrm{~Hz}$ (Cone-Wesson and Ramirez ${ }^{12}$; Vander Werff $^{60}$ ), a small correction factor of $10 \mathrm{~dB}$ was added to the WB bone threshold for infants. This was a best estimate, as the exact correction factor for a WB CE-Chirp by bone conduction in infants has not been established. Ferm et $\mathrm{al}^{28}$ found large corrections of $30 \mathrm{~dB}$ and $20 \mathrm{~dB}(500$ and $1000 \mathrm{~Hz})$ for bone conduction relative to air conduction thresholds in infants. The discrepancy may be due to the supra-aural earphones used for air conduction in their study rather than the insert phones preferred in the United States.

To determine thresholds in eHL, the correction factors from - Table 1 are applied to the observed thresholds to predict the audiogram. When minimum test values are obtained, thresholds should be designated as less than or equal to the corrected value.

\section{Auditory Neuropathy (AN) Screening}

A routine screening for AN can be included in the protocol for testing of children. At first, a moderate level $(\sim 80 \mathrm{~dB} \mathrm{nHL})$ click should be used to generate an ABR. Most systems allow for an alternating polarity stimulus and will divide the rarefaction and condensation averages for display afterward. Averaging should be continued until the noise is less than 50 $\mathrm{nV}$ to insure a quiet recording. Next, display the response in the polarity split mode and examine for a cochlear microphonic which presents as a mirror image starting in the first millisecond after stimulation (Starr et $\mathrm{al}^{53}$ ). Finally, proceed based on local standards for diagnosis and management of auditory neuropathy.

\section{ASSR-Recommended Procedures}

The ASSR procedure should begin with the same preliminary child preparation, electrodes, and WB CE-Chirp threshold search as previously described. The appropriate stimulus rate setting for children is $90 \mathrm{~Hz}$. The default stimuli are NB CE-Chirps. The test method priority should be set to "speed." As with ABR, the "speed" setting will provide a response that is slightly less strict (closer to 95\% confidence) than the "accuracy" setting.

In most cases, both ears will be tested simultaneously. If a unilateral or significant asymmetric loss is suspected, the ears should be tested individually with the better ear tested first. This possibility can be determined during the initial WB chirp test. Contralateral masking is available if necessary. As with $A B R$, it is prudent to set each of the frequencyspecific stimuli in each ear at 0-20 dB greater than the WB CE-Chirp threshold for that ear to begin the test. An AR level of $40 \mathrm{nV}$ is an appropriate starting level for children. This level can be increased during testing if a child is particularly noisy.

It is prudent to watch the background EEG and wait for the child to settle before testing starts. Once the child is quiet, the START button will start all stimuli at once. All eight responses will be tracked at the same time.

The tester must watch the entire screen. The response window for each test looks much like the $F_{\mathrm{MP}}$ graph. The noise is shown as a shadow and falls off substantially with averaging. The response trace, tagged with the associated stimulus level, will grow with averaging if a response is present and will stop the average and change from red to green when the response criteria are met. The actual values of the response criteria percentage and the background noise can be read at any time from a table at the bottom of the screen.

If not stopped by a detected response, the averaging continues for six minutes. There is no automatic stopping for low noise presently implemented in the Eclipse software. The Sininger et $\mathrm{al}^{50}$ study allowed testers to manually stop an average if all the following conditions were met: (a) the response criterion measure was $50 \%$ or less, (b) the background noise was $\leq 15 \mathrm{nV}$, and (c) at least three minutes had elapsed. This procedure was used to shorten the test time. As with $A B R$, the user can extend the averaging time if it appears that a response is growing and is approaching a positive response.

When a response is reached at a given level, the next test level is chosen with a mouse click inside the appropriate response box, and acquisition will start up immediately. Consequently, many different levels may be running simultaneously. However, the system will alert the user if the level of any one stimulus differs from any other simultaneous stimulus by $30 \mathrm{~dB}$ or more. The system will not allow a large discrepancy in the presentation of stimulus levels to avoid the spread of masking. A disallowed level is placed in a "waiting" mode and will start up when the levels of other stimuli allow.

As with $F_{\mathrm{MP}}$, the length of time needed to achieve a response will be related to the sensation level of the eliciting 
Table 2 Average Hearing $(0.5,1,2$, and $4 \mathrm{kHz}$ ) Thresholds in $\mathrm{dB}$ eHL determined by Electrophysiology (ABR, ASSR or Both) for all 10 Participants (P1-10) from Sininger et $\mathrm{al}^{50}$ in Whom DPOAEs Were Absent at all Frequencies and WB Tympanometry Was Found to be Normal

\begin{tabular}{|l|l|l|l|l|l|l|l|l|l|l|}
\hline & P1 & P2 & P3 & P4 & P5 & P6 & P7 & P8 & P9 & P10 \\
\hline RE Avg & 100 & 97.5 & 95 & 90 & 80 & 77.5 & 70 & 47.5 & 36.25 & 28.75 \\
\hline LE Avg & 100 & 100 & 95 & 90 & 43.75 & 78.75 & 65 & 18.75 & 36.25 & 17.5 \\
\hline Bone & $>50$ & $>50$ & & & $>50$ & $>50$ & & & & \\
\hline
\end{tabular}

Note: Bone conduction was tested by WB CE-Chip in four subjects.

stimulus and the level of the background noise. The responses that are confirmed in less than one minute are the most robust and vice versa. This information can be useful in choosing levels in the threshold bracketing process. The ASSR system will automatically plot the uncorrected and corrected (based on - Table 2) thresholds onto an "estimated" audiogram form as shown in the case studies.

\section{Case Studies}

Two cases from Sininger et $\mathrm{al}^{50}$ study have been chosen to illustrate the protocol. The first case involves a one-monthold infant who failed newborn hearing screening but had no other risk factors associated with hearing loss. The infant was evaluated during natural sleep. Test results from this baby are found in - Figures $\mathbf{4 A}$ and $\mathbf{B}$. The middle ear measurements and DPOAE results are within normal limits in both the ears.

It should be noted that the 226-Hz tympanogram was routinely extracted but is not appropriate for a one-monthold infant. The electrophysiologic tests for this baby are shown in - Figure 4B. ABR was used to obtain responses $\left(F_{\mathrm{MP}}>2.25\right)$ to WB CE-Chirps at $10 \mathrm{~dB}$ in each ear. Thresholds were obtained at all four frequencies in each ear for each test.

For both ABR and ASSR, the lowest level tested was $20 \mathrm{~dB}$ at $500 \mathrm{~Hz}$ and $10 \mathrm{~dB}$ at all other frequencies. In this case, ASSR testing was started at $20 \mathrm{~dB}$, and the lowest levels produced a response at all frequencies in the right ear but were elevated by $5 \mathrm{~dB}$ at 500 and $1000 \mathrm{~Hz}$ in the left. The ABR results are shown for 1000 and $4000 \mathrm{~Hz}$ as examples. Testing started at $20 \mathrm{~dB}$ in the left ear, but only the lowest test level $(10 \mathrm{~dB})$ was evaluated on the right. The strategy of only testing the base level was often used when such encouraging results were obtained in preliminary tests and this saved time. ABR responses were determined using the $F_{\mathrm{MP}}$ criteria of 2.25 . The corrected thresholds are shown on the predicted audiogram at the bottom of $\mathbf{- F i g u r e ~} \mathbf{4 B}$. Both techniques found the same results of $0-5 \mathrm{~dB}$ thresholds. The time needed to complete the ASSR testing was 9.5 minutes, and the ABR was completed in 9.3 minutes.

The second case is presented in - Figures $\mathbf{5 A}$ and $\mathbf{B}$. This three-month-old failed NHS after an emergency C-section birth. Two other audiologic assessments had been carried out before this infant was enrolled in the study. The first of these assessments found a mild, likely sensorineural hearing loss and the second ABR again showed a mild, but possibly conductive loss. The tympanometry on the day of this assessment showed peaked tympanograms in both ears with normal middle ear pressure in the right and -300 daPa in the left ear. The absorbance, when corrected for middle ear pressure, was primarily within the normal range but displayed absorbance peaks slightly greater than normal at $\sim 1500$ and $4000 \mathrm{~Hz}$ in both ears. The DPOAEs were considered absent at all frequencies in both ears.

The WB CE-Chirp thresholds were $50 \mathrm{~dB}$ in the right ear and $45 \mathrm{~dB}$ on the left (uncorrected). ASSR threshold searches started around $60 \mathrm{~dB}$, and the final thresholds ranged from 45-55 in the right to 30-55 in the left ear. The corrected puretone averages by ASSR were $41.25 \mathrm{~dB}$ eHL in the right and $35 \mathrm{~dB}$ eHL in the left ear.

ABR thresholds were generally consistent with the ASSR but were slightly higher with PTAs of $48.75 \mathrm{~dB}$ eHL in the right and $43.75 \mathrm{~dB}$ eHL in the left ear. In general, thresholds by ABR were slightly higher than those found with ASSR by Sininger et al. ${ }^{50}$ Sound-field behavioral thresholds were also evaluated and found to be 51.25-dB HL on average, consistent with sound field to insert earphone correction factors.

Finally, WB CE-Chirp thresholds were obtained by unmasked bone conduction revealing thresholds of 30 on the right and 35 on the left. These can be compared with 50 (R) and $45(\mathrm{~L}) \mathrm{dB}$ (dial) by air conduction. Bone-conduction thresholds are expected to be lower than air-conducted thresholds in infants without conductive hearing loss, but the correction from air to bone for a WB stimulus is unclear. If the initial assumption of about $10 \mathrm{~dB}$ holds up, it appears that there is a possible $10 \mathrm{~dB}$ air-bone gap on the right and no appreciable gap on the left.

Only the 500 and $2000 \mathrm{~Hz}$ ABRs are shown, but thresholds were found for all four frequencies. The evaluation time for this infant by ABR (excluding WB air and bone) was 43.98 minutes. The ASSR thresholds were obtained in 32.57 minutes. Overall, faster evaluations by ASSR than ABR were commonly found by Sininger et al. ${ }^{50}$

\section{Discussion}

The purpose of this publication is to provide all details of the testing protocol that produced audiometric predictions on children with reduced test times. This is provided to assist clinicians seeking to replicate the findings in Sininger et $\mathrm{al}^{50}$ so that they can use the same procedures. 
A 1 Month-old, Natural Sleep, Failed NHS, No Other Risks

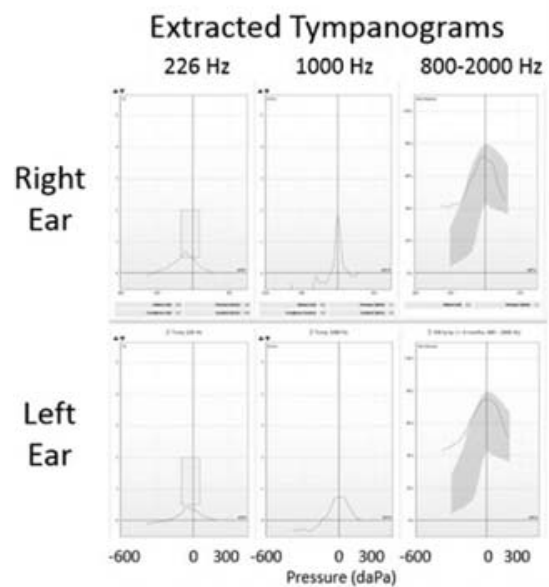

\section{Wide Band Absorbance}

DP-Gram

B
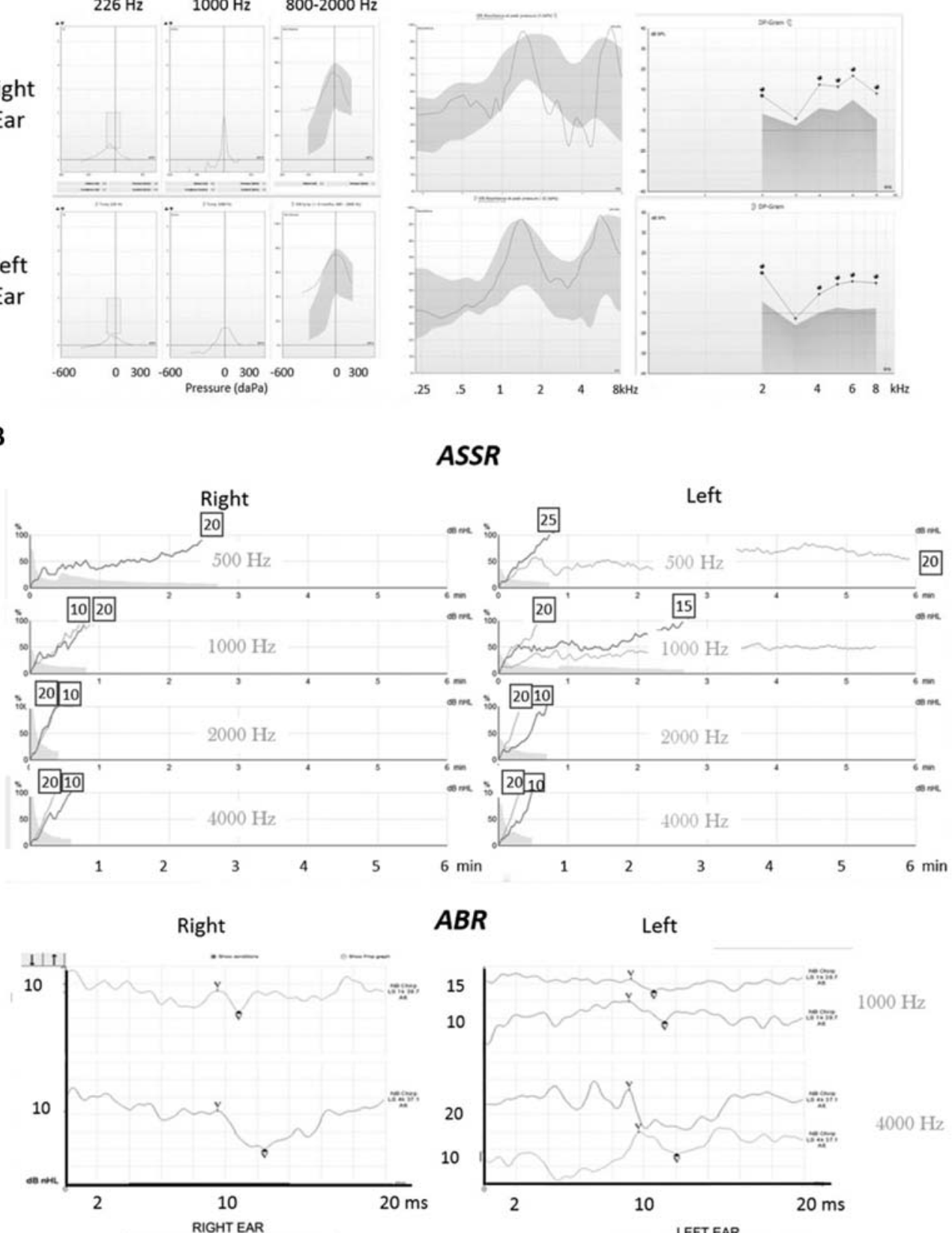

ABR

ASSR

Left
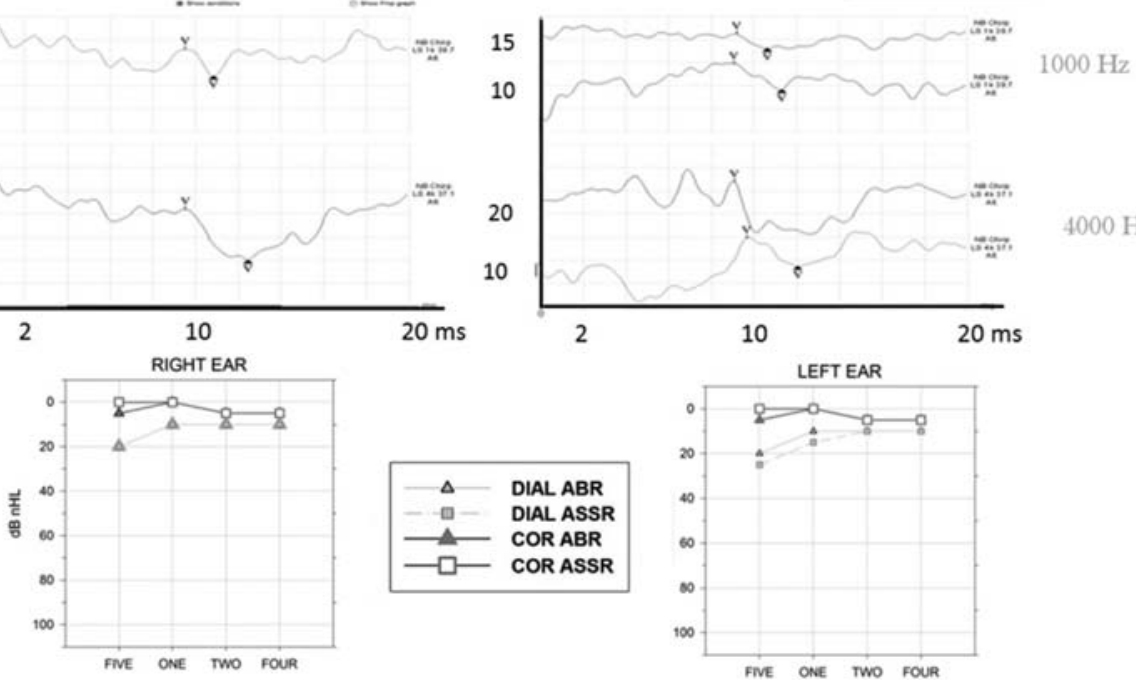

Fig. 4 (A) Case 1. Tympanometry and distortion-product otoacoustic emissions results on Case 1. Tympanograms were extracted from the click response at $226 \mathrm{~Hz}, 1000 \mathrm{~Hz}$ and a composite tympanogram from 800 to $2000 \mathrm{~Hz}$. The latter is superimposed on normative data for a onemonth-old infant. The extracted tympanograms showed normal middle ear pressure and absorbance peaks within the normal range. In the center, the absorbance at peak pressure ( $4 \mathrm{daPa}$ in the right and $-32 \mathrm{daPa}$ in the left ear) is plotted by frequency. Bothears show data that is superimposed upon, and essentially within the norms fora 1-month old child. On the right panels are DPOAE results. Clear responses were noted at all frequencies except $3 \mathrm{kHz}$ in both ears. (B) Electrophysiologic results from Case 1. The ASSR was completed at all four frequencies in each ear as illustrated above. The uncorrected thresholds for each are plotted on the audiogram at the bottom. All ABR uncorrected thresholds are also shown on the audiogram, and the actual averages for 1000 and $4000 \mathrm{~Hz}$ are illustrated above. Thresholds predicted after corrections (COR) are also shown for both technologies and indicate normal hearing sensitivity. 


\section{Months Old-Failed NHS- Third Test- Natural Sleep}

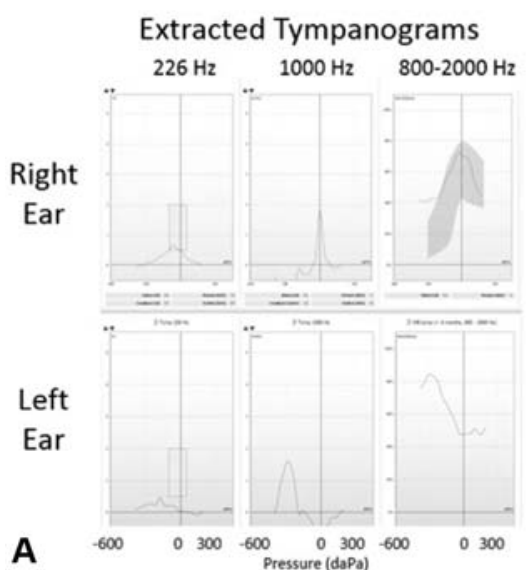

Wide Band Absorbance

DP-Gram
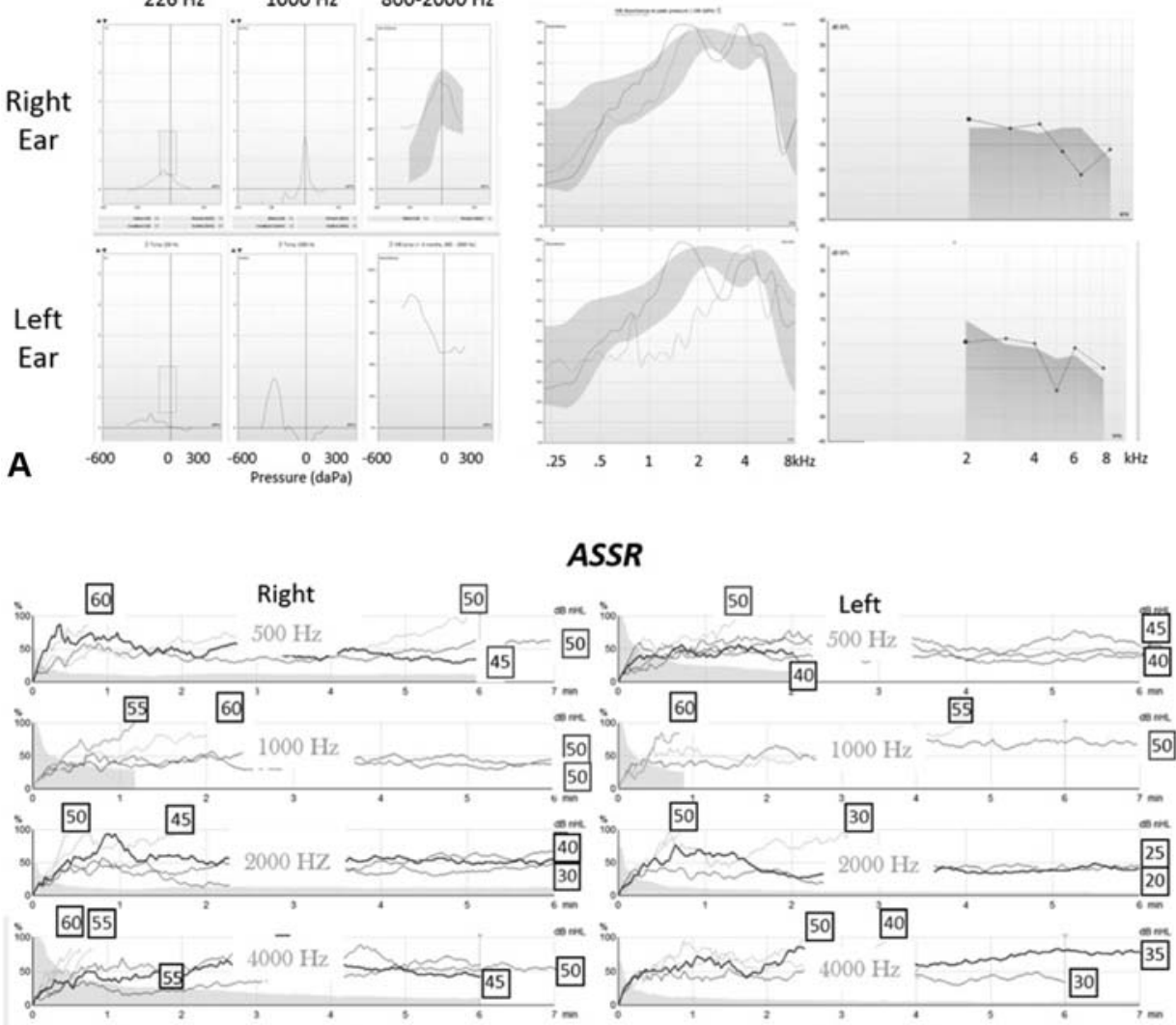

B
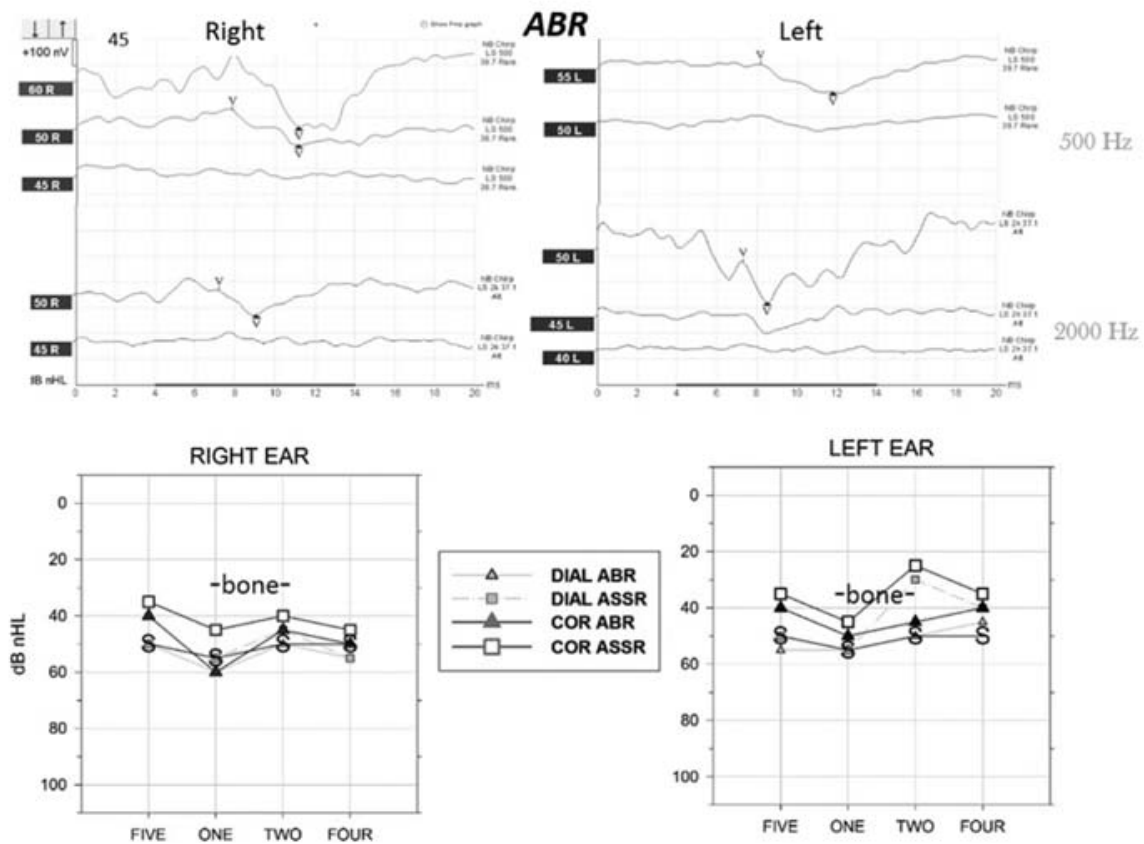

Fig. 5 (A) Tympanometry and distortion-product otoacoustic emissions results on Case 2. Tympanograms from $226 \mathrm{~Hz}, 1000 \mathrm{~Hz}$ and a composite tympanogram from 800 to $2000 \mathrm{~Hz}$ were extracted from the broad band (click) response. In the center, the Absorbance at 0 daPa and corrected peak pressure ( $-105 \mathrm{daPa}$ in the right and $-298 \mathrm{daPa}$ in the left ear) is plotted by frequency. When corrected for peak pressure, both ears show data that is primarily superimposed upon the norms for a 3-month old child. The extracted tympanograms show normal middle ear pressure in the right ear and negative middle ear pressure in the left ear. Normal amplitude absorbance peaks are seen on the tympanograms in both ears. On the far right are DPOAE results. No response was noted at any test frequency in either ear. (B) Electrophysiologic results from Case 2. The initial WB-CE-Chirp ABR thresholds by air conduction were $50 \mathrm{~dB}$ in the right ear and 45 in the left. The ASSR was completed at all four frequencies in each ear. The thresholds for each are plotted on the audiogram at the bottom. All ABR thresholds are also shown on the audiogram, and the actual averages for 500 and $2000 \mathrm{~Hz}$ are illustrated above. Thresholds predicted after corrections are also shown for both technologies and indicate a moderate flat hearing loss with slightly lower thresholds predicted by ASSR. Also shown are Sound-Field (S) audiometric thresholds. 


\section{Application of "Good Clinical" Principles in Testing}

The suggested protocol uses several principles that are supported by both the need for clinical efficiency and by evidence. One is that the order of the tests can improve efficiency. Knowing the status of the middle ear and the outer hair cells can save valuable time and avoid unnecessary testing. Therefore, as in the Sininger et $\mathrm{al}^{50}$ study, this protocol recommends performing otoacoustic emissions tests and tympanometry (WB if possible) at the beginning of the evaluation.

The second principle is to use, whenever possible, wellestablished technologies to improve the SNR of the electrophysiologic recording. Two examples of such technologies are Bayesian weighting (Elberling and Wahlgreen ${ }^{25}$ ) for control of recording noise and narrowband CE-Chirp stimuli (Sturzebecher) for enhancing the amplitude of the auditory neurophysiologic response.

Another principle of the protocol is to save time in any way that is reasonable. This protocol used the same hardware for tympanometry and otoacoustic emissions: in this case, the Interacoustics Titan. Time was saved by inserting the test probe into each ear just once and changing software twice. For example, tympanometry could be performed in the right ear followed by DPOAE, changing the probe to the left ear, starting with DPOAE and completing tympanometry in the left ear. This not only saved time but also was minimally invasive for the infant.

The final principle of the protocol is to use carefully developed and experimentally proven objective methods for response detection. Both $F_{\mathrm{MP}}$ for ABR or detection by "next-generation" ASSR will meet these criteria. Such techniques will improve accuracy of results as well as speed/ efficiency of the test time.

\section{Optimization on Other Clinical Electrophysiology Systems}

The Sininger et $\mathrm{al}^{50}$ study used the Interacoustics Eclipse system. Many of the types of features that we found useful in improving the quality of recordings and reducing test are implemented on other clinical systems. Most systems allow for a variety of tests to be administered from the same piece of equipment, e.g., OAEs and middle ear measurements with the same probe placement, which saved time and was more convenient. Using one piece of equipment means fitting the correct probe size is easier because only one probe was needed. Combined hardware and software are also more cost efficient and space efficient.

Several systems include measurement of real-time residual noise or even SNR. However, specifics on these calculations are not always published or readily available. It is important to know how noise is calculated and how to interpret the values. It is also important to know whether these calculations are available to the clinician in terms of stopping rules for averaging. Knowing the values after the averaging has stopped is valuable but will not improve the overall testing speed.

Many systems provide reproducibility statistics for waveforms, either replications or split-half waveforms. These measures have long been considered as a proxy for SNR, and they can be useful. However, there are no guidelines to indicate the level of correlation value that is "acceptable" nor is there a window placement or duration for correlation that is recommended.

Users should obtain as much information as possible on the implementation of automated noise or detection schemes on any piece of equipment they use or hope to buy. If none is available, practical measures can be substituted. Watch the ongoing EEG to indicate the levels of noise that are contributing to the response and intervene if they are too high. It is useless to record 2,000 or 4,000 sweeps of noise and hope to interpret the results later! When noise levels are low and stable, watch the average for the desired waveforms to emerge. If possible, check the split halves for consistency and manually stop the averaging when the response is present. A response replication at the lowest level can be helpful when this approach is used.

\section{What was Learned from Preliminary DPOAE and Immittance Tests?}

Although tympanometry will not predict average hearing levels, 3-D tympanometry that is normal on every metric can impact the decision of whether to use clinic time for bone conduction (Hunter et $\mathrm{al}^{32}$; Prieve et $\mathrm{al}^{44}$; Aithal et $\mathrm{al}^{1}$ ). Normal middle-ear measures will also have a significant impact on the interpretation of otoacoustic emissions tests (Blankenship et $\mathrm{al}^{3}$ ).

For example, ten children from the Sininger et $\mathrm{al}^{50}$ study were found to have "normal" WB tympanometry and absent DPOAEs. All these children were subsequently found to have significant hearing loss as shown in - Table 2.

Sininger et $\mathrm{al}^{50}$ found that $\mathrm{ABR}$ (with automated detection as described here) could be expected to predict a full audiogram in both ears of typical infant and toddler patients in less than 33 minutes and ASSR could do the same in less than 20 minutes. Ninety percent of children could be tested in 53.7 minutes or less by ABR and 31.26 minutes by ASSR. It is not necessary to do both tests. Based on the results of Sininger et $\mathrm{al}^{50}{ }^{50}$ either ABR or ASSR will provide accurate results in a reasonable amount of time. The important indication is that these procedures will allow most children to be evaluated during natural sleep by ABR and nearly all by ASSR. The consequence of shorter test times for those children who require anesthesia is also important.

\section{Abbreviations}

$\begin{array}{ll}\text { ABR } & \text { auditory brainstem response } \\ \text { AR } & \text { artifact rejection } \\ \text { ASSR } & \text { auditory steady-state response } \\ \text { DPOAEs } & \text { distortion product otoacoustic emissions } \\ \text { LS } & \text { level specific } \\ \text { SNR } & \text { signal-to-noise ratio } \\ \text { WB } & \text { wideband }\end{array}$

Conflict of Interest

None declared. 
Funding

This study was supported by a grant from the Oticon Foundation.

\section{Acknowledgments}

The authors acknowledge the support of audiologists Erica Schicke AuD, Tamara Scott AuD, Carrie Wingo, AuD, Gayle Riemer MA, Kelly Baroch AuD, Emily Spitzer AuD, Laurel Okulski AuD, Shana Jacobs AuD, Lauren Johnson AuD, Mallory Baker AuD, and Sarah Martinho AuD, and research support staff Amanda Ruiz and Morgan Banberger BS.

\section{References}

1 Aithal S, Kei J, Driscoll C, Khan A, Swanston A. Wideband Absorbance outcomes in newborns: a comparison with high-frequency tympanometry, automated brainstem response, and transient evoked and distortion product otoacoustic emissions. Ear Hear 2015;36(05):e237-e250

2 Aithal V, Kei J, Driscoll C, Murakoshi M, Wada H. Predictive accuracy of sweep frequency impedance technology in identifying conductive conditions in newborns. J Am Acad Audiol 2018;29 (02):106-117

3 Blankenship CM, Hunter LL, Keefe DH, Feeney MP, Brown DK, McCune A, Fitzpatrick DF, Lin L. Optimizing clinical interpretation of distortion product otoacoustic emissions in infants. Ear Hear 2018;39:1075-1090

4 Cebulla M, Elberling C. Auditory brain stem responses evoked by different chirps based on different delay models. J Am Acad Audiol 2010;21(07):452-460

5 Cebulla M, Lurz H, Shehata-Dieler W. Evaluation of waveform, latency and amplitude values of chirp ABR in newborns. Int J Pediatr Otorhinolaryngol 2014;78(04):631-636

6 Cebulla M, Sturzebecher E. Automated auditory response detection: further improvement of the statistical test strategy by using progressive test steps of iteration. Int J Audiol 2015;54(08): 568-572

7 Cebulla M, Sturzebecher E, Elberling C. Objective detection of auditory steady-state responses: comparison of one-sample and q-sample tests. J Am Acad Audiol 2006;17(02):93-103

8 Cebulla M, Sturzebecher E, Elberling C, Muller J. New clicklike stimuli for hearing testing. J Am Acad Audiol 2007;18(09): 725-738

9 Cho SW, Han KH, Jang HK, Chang SO, Jung H, Lee JH. Auditory brainstem responses to $\mathrm{CE}-\mathrm{Chirp}(\mathrm{R})$ stimuli for normal ears and those with sensorineural hearing loss. Int J Audiol 2015;54(10): 700-704

10 Cobb KM, Stuart A. Auditory brainstem response thresholds to airand bone-conducted CE-chirps in neonates and adults. J Speech Lang Hear Res 2016;59(04):853-859

11 Cobb KM, Stuart A. Neonate auditory brainstem responses to CEchirp and CE-chirp octave band stimuli I: versus click and tone burst stimuli. Ear Hear 2016;37(06):710-723

12 Cone-Wesson B, Ramirez GM. Hearing sensitivity in newborns estimated from ABRs to bone-conducted sounds. J Am Acad Audiol 1997;08(05):299-307

13 de Boer E. A cylindrical cochlea model: the bridge between two and three dimensions. Hear Res 1980;03(2):109-131

14 Don M, Elberling C. Evaluating residual background noise in human auditory brain-stem responses. J Acoust Soc Am 1994; 96(05 Pt 1):2746-2757

15 Don M, Elberling C. Use of quantitative measures of auditory brain-stem response peak amplitude and residual background noise in the decision to stop averaging. J Acoust Soc Am 1996;99 (01):491-499
16 Don M, Elberling C, Waring M. Objective detection of averaged auditory brainstem responses. Scand Audiol 1984;13(04): 219-228

17 Dworsak-Dodge M, Gravel J, Grimes A, Hunter L, Johnson K, Neault M, Yellin W. Audiologic Guidelines for the Assessment of Hearing in Infants and Young Children. 2012. www.Audiology.org

18 Elberling C, Callo J, Don M. Evaluating auditory brainstem responses to different chirp stimuli at three levels of stimulation. J Acoust Soc Am 2010;128(01):215-223

19 Elberling C, Don M. Quality estimation of averaged auditory brainstem responses. Scand Audiol 1984;13(03):187-197

20 Elberling C, Don M. Detection functions for the human auditory brainstem response. Scand Audiol 1987;16(02):89-92

21 Elberling C, Don M. Auditory brainstem responses to a chirp stimulus designed from derived-band latencies in normalhearing subjects. J Acoust Soc Am 2008;124(05):3022-3037

22 Elberling C, Don M. A direct approach for the design of chirp stimuli used for the recording of auditory brainstem responses. J Acoust Soc Am 2010;128(05):2955-2964

23 Elberling C, Don M, Cebulla M, Sturzebecher E. Auditory steadystate responses to chirp stimuli based on cochlear traveling wave delay. J Acoust Soc Am 2007;122(05):2772-2785

24 Elberling C, Don M, Kristensen SG. Auditory brainstem responses to chirps delivered by an insert earphone with equalized frequency response. J Acoust Soc Am 2012;132(02):El149-El154

25 Elberling $C$, Wahlgreen 0 . Estimation of auditory brainstem response, ABR, by means of Bayesian inference. Scand Audiol 1985;14(02):89-96

26 Ferm I, Lightfoot G. Further comparisons of ABR response amplitudes, test time, and estimation of hearing threshold using frequency-specific chirp and tone pip stimuli in newborns: findings at 0.5 and $2 \mathrm{kHz}$. Int J Audiol 2015;54(10):745-750

27 Ferm I, Lightfoot G, Stevens J. Comparison of ABR response amplitude, test time, and estimation of hearing threshold using frequency specific chirp and tone pip stimuli in newborns. IntJ Audiol 2013;52(06):419-423

28 Ferm I, Lightfoot G, Stevens J. Provisional stimulus level corrections for low frequency bone-conduction ABR in babies under three months corrected age. Int J Audiol 2014;53(02):132-137

29 Hunter LL, Keefe DH, Feeney MP, Brown DK, Meinzen-Derr J, Elsayed AM, Amann JM, Manickam V, Fitzpatrick D, Shott SR. Wideband acoustic immittance in children with Down syndrome: prediction of middle-ear dysfunction, conductive hearing loss and patent PE tubes. Int J Audiol 2017;56(09):622-634

30 Hunter LL, Keefe DH, Feeney MP, Fitzpatrick DF. Pressurized wideband acoustic stapedial reflex thresholds: normal development and relationships to auditory function in infants. J Assoc Res Otolaryngol 2017;18(01):49-63

31 Hunter LL, Keefe DH, Feeney MP, Fitzpatrick DF, Lin L. Longitudinal development of wideband reflectance tympanometry in normal and at-risk infants. Hear Res 2016;340:3-14

32 Hunter LL, Prieve BA, Kei J, Sanford CA. Pediatric applications of wideband acoustic immittance measures. Ear Hear 2013;34(01): $36 s-42 s$

33 Hyde M, Sininger YS, Don M. Objective detection and analysis of auditory brainstem response: an historical perspective. Semin Hearing 1998;19(01):16

34 Janssen RM, Usher L, Stapells DR. The British Columbia's Children's Hospital tone-evoked auditory brainstem response protocol: how long do infants sleep and how much information can be obtained in one appointment? Ear Hear 2010;31(05):722-724

35 Jerger J, Hayes D. The cross-check principle in pediatric audiometry. Arch Otolaryngol 1976;102(10):7

36 Joint-Committee-On-Infant-Hearing. Year 2007 position statement: priniciples and guidelines for early hearing detection and intervention programs. Pediatrics 2007;120:23

37 Korczak P, Smart J, Delgado R, Strobel TM, Bradford C. Auditory steady-state responses. J Am Acad Audiol 2012;23(03):146-170 
38 Kristensen SG, Elberling C. Auditory brainstem responses to levelspecific chirps in normal-hearing adults. J Am Acad Audiol 2012; 23(09):712-721

39 Lightfoot G, Stevens J. Effects of artefact rejection and Bayesian weighted averaging on the efficiency of recording the newborn ABR. Ear Hear 2014;35(02):213-220

40 McCreery RW, Kaminski J, Beauchaine K, Lenzen N, Simms K, Gorga MP. The impact of degree of hearing loss on auditory brainstem response predictions of behavioral thresholds. Ear Hear 2015;36(03):309-319

41 Michel F, Jorgensen KF. Comparison of threshold estimation in infants with hearing loss or normal hearing using auditory steady-state response evoked by narrow band CE-chirps and auditory brainstem response evoked by tone pips. Int J Audiol 2017;56(02):99-105

42 Myers J, Kei J, Aithal S, Aithal V, Driscoll C, Khan A, Manuel A, Joseph A, Malicka AN. Development of a diagnostic prediction model for conductive conditions in neonates using wideband acoustic immittance. Ear Hear 2018;39(06):1116-1135

43 Myers J, Kei J, Aithal S, Aithal V, Driscoll C, Khan A, Manuel A, Joseph A, Malicka AN. Diagnosing middle ear pathology in 6- to 9month-old infants using wideband absorbance: a risk prediction model. J Speech Lang Hear Res 2018;61(09):2386-2404

44 Prieve BA, Vander Werff KR, Preston JL, Georgantas L. Identification of conductive hearing loss in young infants using tympanometry and wideband reflectance. Ear Hear 2013;34(02):168-178

45 Rodrigues GR, Lewis DR. Establishing auditory steady-state response thresholds to narrow band CE-chirps in full-term neonates. Int J Pediatr Otorhinolaryngol 2014;78(02):238-243

46 Sininger YS. Auditory brain stem response for objective measures of hearing. Ear Hear 1993;14(01):23-30

47 Sininger YS. Filtering and spectral characteristics of averaged auditory brain-stem response and background noise in infants. J Acoust Soc Am 1995;98(04):2048-2055

48 Sininger YS, Abdala C, Cone-Wesson B. Auditory threshold sensitivity of the human neonate as measured by the auditory brainstem response. Hear Res 1997;104(1-2):27-38

49 Sininger YS, Grimes A, Christensen E. Auditory development in early amplified children: factors influencing auditory-based communication outcomes in children with hearing loss. Ear Hear 2010;31(02):166-185

50 Sininger YS, Hunter LL, Hayes D, Roush PA, Uhler KM. Evaluation of speed and accuracy of next-generation auditory steady state response and auditory brainstem response audiometry in children with normal hearing and hearing loss. Ear Hear 2018; 39:1207-1223

51 Spivak LG. Spectral composition of infant auditory brainstem responses: implications for filtering. Audiology 1993;32(03): 185-194

52 Stapells DR, Gravel JS, Martin BA. Thresholds for auditory brain stem responses to tones in notched noise from infants and young children with normal hearing or sensorineural hearing loss. Ear Hear 1995;16(04):361-371

53 Starr A, Sininger Y, Nguyen T, Michalewski HJ, Oba S, Abdala C. Cochlear receptor (microphonic and summating potentials, otoacoustic emissions) and auditory pathway (auditory brain stem potentials) activity in auditory neuropathy. Ear Hear 2001;22 (02):91-99

54 Stuart A, Cobb KM. Effect of stimulus and number of sweeps on the neonate auditory brainstem response. Ear Hear 2014;35(05): 585-588

55 Stuart A, Yang EY. Effect of high-pass filtering on the neonatal auditory brainstem response to air- and bone-conducted clicks. J Speech Hear Res 1994;37(02):475-479

56 Sturzebecher E, Cebulla M. Automated auditory response detection: improvement of the statistical test strategy. Int J Audiol 2013;52(12):861-864

57 Sturzebecher E, Cebulla M, Elberling C. Automated auditory response detection: statistical problems with repeated testing. Int J Audiol 2005;44(02):110-117

58 Sturzebecher E, Cebulla M, Elberling C, Berger T. New efficient stimuli for evoking frequency-specific auditory steady-state responses. J Am Acad Audiol 2006;17(06):448-461

59 Van Maanen A, Stapells DR. Multiple-ASSR thresholds in infants and young children with hearing loss. J Am Acad Audiol 2010;21 (08):535-545

60 Vander Werff KR. Accuracy and time efficiency of two ASSR analysis methods using clinical test protocols. J Am Acad Audiol 2009;20(07):433-452

61 Yoshinaga-Itano C. From screening to early identification and intervention: discovering predictors to successful outcomes for children with significant hearing loss. J Deaf Stud Deaf Educ 2003; 08(01):11-30

62 Yoshinaga-Itano C, Sedey AL, Wiggin M, Chung W. Early hearing detection and vocabulary of children with hearing loss. Pediatrics 2017;140(02). doi: 10.1542/peds.2016-2964 